Apidologie, 1977, 8 (4), 369-391.

\title{
FORTSCHRITTE AUF DEM GEBIET DER HONIGTAU-FORSCHUNG
}

\section{Progrès récents dans le domaine de recherche sur le miellat}

\author{
Hartwig KUNKEL und Werner KLOFT \\ Institut für Angewandte Zoologie der Universität Bonn, \\ An der Immenburg 1, 5300 Bonn, BRD
}

\section{SUMMARY}

\section{PROGRESS IN HONEYDEW RESEARCH}

In central Europe bees collect the honeydew from only few (58 known) species of Sternorrhyncha (coccids, aphids, psyllids) (Table 1). This collecting it favored by an aggregation near the floral parts, in roll galls, and at the peripheral branches of trees combined with less visitation by ants. But $72.4 \%$ of all species possess a filter chamber. As a consequence, these aphids secret 2-3 times more honeydew with $25-40 \%$ dry weight sucrose (from coccids and aphids) instead of less than $1 \%$ sucrose found from aphids without a filter - gut. The appearance of other types of carbohydrates is discussed. Trehalose seems to occur in every honey-dew. Since raffinose is known to be widespread as a constituent of phloem sap, its occurence in honeydew is also discussed. Not all the aphids can assimilate raffinose as seen from the artificial diet studies. The honeydew from $M$. persicae is collected by bees, and, on the other hand, these aphids can be reared on artificial diet well. As a model their nutritional physiology can be well studied, and some results are reviewed. At the end, significance of host plants is shown to the following items : summer depression in aphid populations, the analysis of phloem sap, influence of saliva in nutrition.

\section{ZUSAMMENFASSUNG}

Bienen sammeln den Honigtau von nur wenigen (58) einheimischen Pflanzenlaus-Arten (Tab. 1) ein. Begünstigt wird das Sammeln durch Bildung von Aggregationen in der Nähe von Blüten, in Rollgallen, und im peripheren Bereich der Bäume mit geringem Ameisenbesuch. Vor allem aber fällt auf, daB $72,4 \%$ der angeführten Spezies einen Filterdarm besitzen. Als Konsequenz wird erkannt, daß Aphiden eine 2-3 mal höhere Abgabefrequenz bei einem Honigtau haben, in dem 25-40\% (bei Aphiden und Cocciden) anstatt unter $1 \%$ (bei Aphiden ohne Filterdarm) des Trockengewichtes an Saccharose enthalten sind. Das Vorkommen weiterer 
Kohlenhydrate wird diskutiert. Trehalose scheint in jedem Honigtau vorzukommen. Nachdem die weite Verbreitung von Raffinose im Phloem bekannt geworden ist, wird auch ihr Erscheinen im Honigtau diskutiert. Nicht alle Blattläuse können Raffinose assimilieren, wie ein Vergleich auf künstlicher Diät zeigt. Der Honigtau von $M$. persicae wird gelegentlich von Bienen gesammelt. Da sich diese Art gut auf einer künstlichen Diät halten läßt, konnte sie intensiv ernährungsphysiologisch untersucht werden. Hierzu sind einige Ergebnisse mitgeteilt. Am Schluß wird auf die Bedeutung der Wirtspflanzen bei folgenden Fragen hingewiesen : Sommerliche Depression der Pflanzenlaus-Populationen, die Zusammensetzung des Phloemsaftes, die Beeinflussung der Nahrung durch Speichelabgabe.

\section{1. - NUR WENIGE PFLANZENLÄUSE WERDEN VON DER HONIGBIENE AUFGESUCHT}

Es sind nun gut 10 Jahre her, seitdem eine Liste einheimischer Sternorrhyncha veröffentlicht wurde (KLofr, 1966), die Hinweise auf die Spezies enthällt, deren Honigtau nachgewiesenermaßen von Honigbienen gesammelt wird. Außer einer Bearbeitung der wissenschaftlichen Namen — die deutschen Namen bewähren sich — brauchte die Liste nur wenig verändert zu werden. Neue Haupt-Honigtau-Lieferanten dürften in Mitteleuropa kaum entdeckt werden (s. Tab. 1) Es kommen sicher immer wieder neue Spezies hinzu, die nur gelegentlich Honigtau liefern, wenn die Honigbiene z.B. beim Blütenbesuch darauf stößt.

Als typisches Beispiel sei auf eine Mitteilung von Maurizio und Grafl (1969) hingewiesen, wonach bei der Ackerbohne und Luzerne während der Blütezeit gleichzeitig Nektar und Honigtau an der gleichen Pflanze gesammelt werde (Erzeuger vielleicht Aphis-Arten).

Auch bei der etwas bedeutenderen Honigtautracht von Periphyllus acericola (WALKER) beobachtete Fossel (1963, hier als P. villosus (HARTIG) Synonym zu $P$. testudinaceus (FERNIE) bestimmt), daß die Bienen nur von der Ahornblüte angelockt werden, und « bald Honigtau, bald Nektar aufnehmen, wenn sie die hängenden Blüten von oben her anfliegen ».

Aber gerade die Haupttracht-Lieferanten werden nicht über die Blüten gefunden. Hier scheinen andere Faktoren bestimmend zu sein. Möglicherweise hat dabei das Angebot einer besonders hohen Menge von Honigtau pro Zeiteinheit und Individuum eine Bedeutung, weiterhin die Zusammensetzung an Kohlenhydraten (s.u.).

So kann sich der Honigtau z.B. über Nacht in Rollgallen sammeln, wobei diese wäßrige Lösung häufig weitgehend vor dem Verdunsten bewahrt wird, ohne daß der Zugang völlig gesperrt wäre. Nimmt man einige Spezies (Psylla sp. sp., Phyllaphis fagi (Linnaeus)) heraus, die besser zur nachfolgenden Kategorie gezählt werden sollten, so gehören zu diesen Rollgallen-Bildnern aber nur $8(13,8 \%)$ der in Tabelle 1 aufgeführten 58 Arten.

Mitglieder dieser Kategorie werden aber anscheinend nur gelegentlich örtlich von Bienen besucht. 
TAB. 1. - Europäische Sternorrhyncha, bei denen ein Bienenbeflug eindeutig nachgewiesen ist. $\mathrm{R}$ : Erzeuger einer Rollgalle; F : Filterdarm vorhanden.

TABL. 1. - Sternorrhynches européens visités par les abeilles.

$\mathrm{R}$ - producteur d'une galle

$\mathbf{F}=$ présence d'une chambre filtrante

RF Psylla mali Schmidberger
RF Psylla piri Linneaus
RF Psylla piricola Förster
RF Psylla pyrisuga Förster
RF Psylla crataegi Schrank
RF Psylla ulmi Förster

Psyllina

Coccina

Kermes quercus (Linnaeus)

Eulecanium corni (Bouché)

Eulecanium fletcheri (Cockerell)

Eulecanium rufulum (Cockerell)

Physokermes piceae (Schrank)

Physokermes hemicryphus (Dalman)

\section{Aphidina}

(Nomenklatur nach EAstop und Hille Ris Lambers, 1976)

R Mindarus abietinus Koch

$\mathbf{R}$ Eriosoma (Schizoneura) ulmi (Linnaeus)

R Prociphilus fraxini (Fabricius)

R Prociphilus bumeliae (Schrank)

? Thelaxes dryophila (Schrank)

Pterocomma salicis (Linnaeus)

Periphyllus coracinus (Koch)

Periphyllus acericola (Walker)

Chaitophorus populeti (Panzer)

F Schizolachnus pineti (Fabricius)

F Cinara pinea (Mordvilko)

F Cinara neubergi (Arnhart)

F Cinara brauni Börner

F Cinara cuneomaculata (del Guercio)

F Cinara pilicornis (Hartig)

F Cinara stroyani (Pasek)

F Cinara picea (Panzer)

F Cinara laricis (Hartig)

F Cinara cembrae (Seitner)

F Cinara pini (Linnaeus)

F Cinara pectinatae (Nördlinger)

F Cinara confinis (Koch)

F Cinara cupressi (Buckton)

F Cinara juniperi (de Geer)

F Cinara costata (Zetterstedt)

F Taberolachnus salignus (Gmelin)

F Lachnus roboris (Linnaeus)

F Lachnus pallipes (Hartig)

F Clethrobius giganteus (Cholodkovsky)

F Phyllaphis fagi (Linnaeus)

F Betulaphis quadrituberculata (Kaltenbach)

F Callaphis juglandis (Goeze)

F Pterocallis maculata (von Heyden)

F Myzocallis carpini (Koch)

F Myzocallis coryli (Goeze)

F Myzocallis castanicola Baker

F Tuberculatus querceus (Kaltenbach)

F Tuberculatus (Tuberculoides) annulatus (Hartig)

F Eucallipterus tiliae (Linnaeus)

Hyalopterus pruni (Geoffroy)

Hyalopterus amygdali (Blanchard)

R Aphis pomi de Geer

$\mathrm{R}$ Brachycaudus cardui (Linnaeus)

R Brachycaudus (Appelia) schwartzi (Börner)

R Myzus persicae (Sulzer)

(R) Myzus cerasi (Fabricius)

(Bisher unbekannte Aphididae an Vicia faba, Medicago sativa und Getreide-Arten)

(Aphididae inconnus jusqu'à présent sur Vicia faba, Medicago sativa et les céréales). 
Lieferanten von Haupttrachten befinden sich unter den restlichen Pflanzenläusen, und zwar unter den $72,4 \%$ der in Tabelle 1 genannten Arten, die eine Filterkammer besitzen. Hierin könnte ein besonders wichtiger Schlüssel für eine Erklärung dafür liegen, weshalb Honigtau nur von bestimmten Pflanzenläusen eingesammelt wird. Auffallend ist weiterhin, daß nahezu alle bekannten Honigtau-Lieferanten Holzbewohner sind. Ehe auf diese Dinge näher eingegangen wird, möchten wir hier betonen, daß selbstverständlich noch andere Faktoren die Bedeutung als Honigtau-Lieferanten an die Honigbiene mitbestimmen.

Dies zeigt sich z.B. sehr schön bei den inzwischen von Fossel (1970; 1971 a, b; 1972 a, b, c; etc.) und Scheurer (1966, 1969, 1970, 1971 etc.) besonders intensiv untersuchten Cinara-Arten. Hier können wir verschiedene ökologische Gruppierungen vornehmen. Cinara-Arten im wahrscheinlichen Ausgangszustand der Evolution siedeln am jüngsten Trieb der Koniferen, wenn möglich, am Maitrieb. Sie bilden eine Vielzahl sehr kleiner Kolonien und werden nur sporadisch von Ameisen besucht. Über eine Reihe von Übergangsstufen besiedeln andere ökologische Gruppen immer ältere Baum-Partien bis zum Stamm; die Kolonien (Aggregationen) werden im Laufe der Evolution immer größer und am Ende völlig von den Ameisen abhängig. Die dickere Rinde verlangt längere Stechborsten, was indirekt an der Zahl der Borstenpaare längst der Rinne des letzten Labium-Gliedes abzählbar ist (hierzu Angaben bei Fossel, 1970; Scheurer, 1970). Mit dem Steigern der Trophobiose-Beziehung gehen auch innerhalb der Gattung Cinara die ursprünglich besonders lang ausgebildeten dorsalen Tibienhaare verloren, ebenso das larvale Verhalten, den Kot mit den Hinterbeinen abzuschleudern (K UNKEL, 1973).

Die ursprüngliche Ausgangsgruppe mit C. pilicornis (Hartig), woh] auch C. costata ('Zetterstedt), dann C. pectinatae (Nördinger), C. cuneomaculata (del Guercio) (Syn. C. boerneri Hille Ris Lambers), C.neubergi Arnhart und $C$. pinea (Mordvilko) bilden nun interessanterweise die wichtigsten Haupttracht-Lieferanten innerhalb der Cinara-Arten. Offenbar wirkt sich die periphere Besiedlung der Bäume und der geringe Kontakt zu Ameisen günstig aus.

\section{2. - DIE BEDEUTUNG DES FILTERDARMES VORKOMMEN DES FILTERDARMES}

Ein Kurzschluß zwischen dem vorderen Bereich des Mitteldarmes und einem Teil des Hinterdarmes ist innerhalb der Sternorrhyncha (Pflanzenläuse) mindestens 8 mal unabhängig voneinander erfunden worden (KUNKEL, unpubl.). Dies läßt sowohl auf ein Bedürfnis als auch auf günstige morphologisch-physiologische Vorbedingungen schließen. Eine Hypothese von BERLESE (1893) besagt, daß (Anteile der) Kohlenhydrate und Wasser über die Filterkammer schnell durch den Körper hindurch geschleust werden, während ein 
nun Stickstoff-angereicherter Anteil des Nahrungssaftes “ den langen Weg » durch den Mitteldarm nimmt. Nach unseren physiologischen Erfahrungen wird man zu diesem “ langen Weg » auch den Bereich der Hämolymphe hinzuzählen müssen.

Munk (1968 a, b, c) konnte die Berlese Hypothese bei Auchenorrhyncha experimentell bestätigen. Er vermutete aufgrund elektronenoptischer Bilder eine Druckfiltration, wobei Aminosäuren zurückgehalten werden, dagegen Saccharose, vielleicht sogar Raffinose, und Azorubin-S (Munk, 1968 b) hindurchgelassen wird. Während ursprünglich alle Cocciden eine Filterkammer besaßen und sie nur als Lokalbibitoren verloren (KuNKEL, 1966, 1967), ist die Filterkammer bei den Aphiden sporadisch verbreitet (Börner, 1938). Unter den in der Liste KLOFT 1966 aufgezählten Spezies gehören Lachniden und Callaphididen (sensu Börner) dazu.

Allerdings verneint Börner (1938, 1952) das Vorhandensein einer Filterkammer für eine Gruppe seiner Callaphididen, die sehr heterogenen Phyllaphidinae (Börner). Für die anderen Mitglieder der Familie beschreibt er folgende ( primitive Filterkammer ) : Der durch einen sehr langen Oesophagus an das Tierende gelangte Beginn des Mitteldarmes liegt ( festgeklebt » auf dem Endteil des Hinterdarmes dicht vor dem After. Dies konnte durch eine Reihe eigener Präparationen bestätigt werden.

Probeweise Untersuchungen (KUNKeL, unpubl.) ergaben aber auch ähnliche Verhältnisse bei Mitgliedern der Unterfamilie Phyllaphidinae Börner. Bei Clethrobius comes (WALKER) ist der Oesophagus nicht ganz so lang; ein längerer Abschnitt des vorderen Mitteldarmes einschließlich der ersten Schleife liegt auf dem Rectum (Hinterdarm) “ festgeklebt ». Drepanosiphum platanoidis (Schrank) hat dagegen ebenfalls einen äußerst langen Oesophagus; aber anders als bei den typischen Callaphididen liegt der Mitteldarm-Beginn frei, ein darauf folgender längerer Mitteldarm-Abschnitt aber festgeklebt auf dem Rectum auf. Diese Filterdärme erinnern stark an jene der primitiven Lachniden (Eulachnus sp. sp.). Daß sie trotz der morphologischen Einfachheit eine besondere physiologische Funktion ausüben, soll folgend dargestellt werden.

\section{Frequenzen der Honigtau-Abgabe bei Aphiden}

Die in Tabelle 2 aus den verschiedensten Quellen kompilierten Zahlen über die Frequenz der Honigtau-Abgabe sind mit großer Vorsicht zu betrachten und sollen nur Tendenzen aufzeigen. Gerade die Frequenz (nicht das Volumen) erweist sich nach Untersuchungen von KunkeL und Hertel (1976, Myzus persicae (Sulzer) auf künstlicher Diät) als ein empfindlicher Indikator für jegliche Veränderungen von Einflüssen auf Blattläuse. Als 
TAB. 2. - Abgabefrequenz (pro Std, und Individuum, möglichst um $20^{\circ} \mathrm{C}$ ) von Honigtau bei verschiedenen Aphiden-Species. 1 Kategorie, O ohne, F mit Filterkammer, Kr Kraut, Ho Holzgewächs. 2 Species. 3 Wirtspflanze. 4 Frequenz, wenn möglich, ausdrücklich während einer kontinuierlichen Phase : (k). 5 Autor.

TABL. 2. - Fréquence de sécrêtion du miellat (par heure et par individu, autant que possible aux alentours de $20^{\circ} \mathrm{C}$ ) chez différentes espèces d'A phidés.

1 : Catégorie $O=$ sans chambre filtrante

$\mathbf{F}=$ avec chambre filtrante

$\mathrm{Kr}=$ plante herbacée

Ho = plante ligneuse

2 : Espèce

3 : Plante hôte

4 : Fréquence, au cours d'une phase continue si possible (k)

5 : Auteurs

\begin{tabular}{|c|c|c|c|c|}
\hline 1 & 2 & 3 & 4 & 5 \\
\hline $\mathrm{O} \mathrm{Kr}$ & Aphis fabae Scopoli & Vicia & 1,46 & Henzig 1938 \\
\hline $\mathrm{O} \mathrm{Kr}$ & " $>\quad$-Larven & Vicia & $0,88-1,5$ & BANKs und Nixon 1959 \\
\hline $\mathrm{O} \mathbf{K r}$ & Schizaphis graminum (Rondani) & $\begin{array}{l}\text { Weizen, } \\
\text { blé } \\
\text { Gerste } \\
\text { orge }\end{array}$ & $1,19-1,6$ & Maxwell und Painter 1959 \\
\hline $\mathrm{O} \mathbf{K r}$ & Myzus persicae (Sulzer) & Raphanus & $1,13-1,4$ & MittLer 1970 \\
\hline $\mathrm{O} \mathbf{K r}$ & Acyrthosiphon pisum (Harris) & Pisum & $\begin{array}{l}1,3-1,4 \\
(\mathrm{k})\end{array}$ & AUCLAIR 1959 \\
\hline $\mathrm{O} \mathbf{K r}$ & Megoura viciae (Buckton) & Vicia & $\begin{array}{l}0,67-0,89 \\
(k)\end{array}$ & EHRHARDT 1963 \\
\hline $\mathrm{O} \mathrm{Ho}$ & Hyalopterus pruni (Geoffroy) & $\begin{array}{l}\text { Prunus } \\
\text { klein, } \\
\text { eingetopft } \\
\text { petit, en pot }\end{array}$ & $\begin{array}{l}2,7 \\
(\mathrm{k})\end{array}$ & SмrTtн 1937 \\
\hline O Ho & Aphis sambuci Linnaeus & Sambucus & 2,18 & Henzig 1938 \\
\hline $\mathbf{F} \mathbf{K r}$ & Therioaphis trifolii (Monell) & $\begin{array}{l}\text { Medicago } \\
\text { sativa }\end{array}$ & $1,67-3,0$ & $\begin{array}{l}\text { MittLer } \\
\text { und SyLvester } 1961\end{array}$ \\
\hline F Ho & $\begin{array}{l}\text { Cinara cuneomaculata } \\
\text { (del Guercio) }\end{array}$ & $\begin{array}{l}\text { Larix- } \\
\text { Zweig im } \\
\text { Zimmer } \\
\text { branches } \\
\text { en chambre }\end{array}$ & $6,0-7,5$ & Fossel 1956 \\
\hline F Ho & Cinara pinea (Mordvilko) & $\begin{array}{l}\text { Pinus } \\
\text { mit Ameisen } \\
\text { avec fourmis }\end{array}$ & $6-9$ & SCHEURER 1971 \\
\hline F Ho & Cinara pini (Linnaeus) & $»$ & 8 & $»$ \\
\hline F Ho & Cinara escherichi (Börner) & $»$ & 8 & $»$ \\
\hline F Ho & Cinara pectinatae (N dlinger) & Abies & $4-6$ & LEONHARDT 1940 \\
\hline F Ho & Tuberolachnus salig :(Gmelin) & Salix & $2,2-6,9$ & MitTLER 1958 \\
\hline
\end{tabular}


Beispiele seien Temperatur, Licht, Luftfeuchte, Luftbewegung, Nahrungsqualität und Nähe eines Koloniemitgliedes genannt. Weiterhin ändert sich die Frequenz im Laufe der nachembryonalen Ontogenie, wobei (außer im unnittelbaren Bereich der Ecdysis) durch Häutungen der allgemeine Verlauf nicht verändert wird. Dennoch scheint jede Spezies bei gleicher Temperatur und auf ihr günstigen Wirtspflanzen unter normalen Verhältnissen einen bestimmten Frequenzbereich einzuhalten. Es wäre in diesem Zusammenhang dringend zu wünschen, eine Aphide mit Filterdarm auf eine künstliche Diät zu bokommen (s. KUNKEL, 1977).

Bei aller Vorsicht also lassen sich aus Tabelle 1 folgende Tendenzen ablesen : 1. An einem Holzgewächs wird häufiger gekotet als an Kraut. 2. Aphiden mit Filterdarm koten 2-3 mal häufiger als solche ohne Filterdarm.

In diesem Zusanmenhang sollten die Gross Growth Efficiency (GGE)Zahlen von Dixon $(1971,1975)$ diskutiert werden. Sie geben eine Energiebilanz (in Kalorien) wieder, wobei der Gewinn an Wachstum in Prozent der aufgenommenen Nahrung ausgedrückt wird. Für Aphiden auf Holzgewächsen erhält er den Bereich 5-20, für jene auf Kraut 43-65 (Dixon, 1975). Ein besonders interessantes Beispiel ergibt sich für Rhopalosiphum padi (LINN $A_{\perp} \mathrm{EUS}$ ), bei der er (1971) auf Prunus padus 8-20, auf Avena sativa die Zahl 43 crhält. Dieses Ergebnis ist sehr interessant, wenn auch einige Bedenken angeroldet werden müssen.

So berücksichtigt er nicht die Verschiedenheiten der ontogenetischen Stadien (Kunkel und Hertel 1975, Hertel und Kunkel, 1976); er mißt die Respiration bei einer anderen Temperatur; aus seinen Werten für Prunus padus errechnen wir die Zahl 26,5.

Grundsätzlich wäre aber zu diskutieren, ob Aphiden an Holzgewächs und Kraut, vor allem aber Aphiden mit und ohne Filterkammer auf diese einfache Art verglichen werden sollten. Der mehr oder weniger große Durehfluß allein von Kohlenhydraten bestimmt die Bilanz. Zumindestens ist der Schluß (Dixon, 1975), daß man indirekt aus diesen Zahlen vermuten könne, die Amino-Stickstoff-Konzentration sei im Phloem von Kräutern höher, anfechtbar.

Für Myzus persicae (Sulzer) können wir bei etwa gleicher HonigtauAbgabefrequenz wie auf Kraut (MitTlen, $1970: 1,1-1,39$; KunKel und Hertel, 1976 : 0,9-1,4 im Durchschnitt etwa 1,1) aus der Energiebilanz bei Kunkel und Hertel (1975) folgende GGE-Zahlen errechnen : alatomorphe L3 17, für apteromorphe L3 35.

\section{Das Vorkommen von Saccharose im Honigtau}

Bei den bisherigen Honigtau-Analysen fällt im Hinblick auf Kohlenhydrate auf, daß von Cocciden und Aphiden mit Filterdarm Saccharose in 
beträchtlichen Mengen ausgeschieden wird. Von einigen Autoren ist sogar etwas voreilig geschlossen worden, daß in diesem Fall eine Invertase $(\alpha-$ Glucosidase) im Darmtrakt fehlen würde. Wie die in Tabelle 3 kompilierten Werte zeigen, liegt der Saccharose-Gehalt bei Filterdarm-Tieren zwischen $25-40 \%$ des Gesamt-Trockengewichtes, während jener bei Läusen ohne Filterdarm unter $1 \%$ liegt. Möglicherweise hat dieses Saccharose-Angebot einen besonderen Wert für die Anlockung von Honigbienen.

Ehe physiologische Konsequenzen auf die Funktion eines Filterdarmes gezogen werden, sei auf die Tatsache verwiesen, daß Saccharose nicht bei allen Filterkammersystemen im abgegebenen Honigtau erscheint. SchefER Immex (1957) fand bei der Zikade Idiocerus laminatus Flon. weder Monosaccharide noch Saccharose, sondern anscheinend nur transglukosidierte Zucker (möglicherweise hat sie Trehalose übersehen, s. unten). KunkeL (unpubl.) konnte durch besondere Techniken in der Dünnschicht-Chromatographie im Kot der Aleyrodine Trialeurodes vaporariorum (Westwood) außer Trehalose nur 13 Flecken höherer Zucker entdecken. Dies erklärt z.T. wohl, daß seit Weber (1931) den Weißen Fliegen ein Kot ohne Kohlenhydrate zugesprochen wird, was natürlich von der Nahrungsquelle her gesehen unwahrscheinlich wäre. Ein ähnliches Bild (in geringer Konzentration Monosaccharide, eine hohe Anzahl verschiedenster Oligosaccharide) ergibt sich übrigens aus der Analyse von Honigtauen diverser (filterdarmloser) Adelgiden (KunKEL, 1966; unpubl.). Hier ist es erklärlich, da der Darm-Durchfluß bei diesen Lokalbibitoren (KUNKEL, 1967) sehr langsam ist. Transglukosidierungen dürften sich übrigens auch bei der vorläufigen Aufbewahrung des Honigtaues in einer Wachshülle (KunKeL, 1972) oder in einer feuchten Galle fortsetzen.

\section{Spekulation über die Leistung einer Filterkammer}

In der Höhe der Honigtau-Abgabefrequenz und der Konzentration von Saccharose reihen sich die primitiven Filterdärme der Callaphididae sensu Börner durchaus ein. Die Cocciden haben eine sehr komplizierte Filterkammer, an welcher der vordere Teil des Hinterdarmes und zusätzlich das Rectum in Form eines Blindsackes beteiligt sind. Außerdem sind die jeweiligen Epithelwände histologisch stark spezialisiert (K UNKEL, 1966).

Setzt man voraus, daß die Trockensubstanz des Phloemsaftes aus etwa $80 \%$ Saccharose besteht, daß die Transport-Vorgänge im Teil des « langen Weges " (Bereich des Mitteldarmes und der Hämolymphe) bei allen Tieren sich in etwa entsprechen, daß weiterhin die Saccharose des Honigtaues fast ausschließlich über den Kurzschluß der Filterkammer geleitet wurde, kann man in erster Näherung folgende Aussage machen : Die Kohlenhydrat-Nahrung (Raffinose wird übrigens auch im Honigtau von Filterdarm-Tieren gefunden, 
TAB. 3. - Angaben über das Vorkommen von Saccharose im Honigtau einiger Cocciden und Aphiden. 1 Kategorie $\mathrm{F}$ mit, $\mathrm{O}$ ohne Filterkammer; 2 Species; 3 Mengenangaben, quantitative Angaben in Prozentzahlen beziehen sich überwiegend auf das Gesamt- Trockengewicht des Honigtaus und sollen nur einen ersten Anhaltspunkt geben. 4 Autoren.

TabL. 3. - Indications sur la présence de saccharose dans le miellat de quelques Coccidés et Aphidés.

1 : Catégorie $\mathbf{F}=$ avec chambre filtrante

$\mathrm{O}=$ sans chambre filtrante

2 : Espèce

3 : Données quantitatives en $\%$. Elles se rapportent principalement au poids sec total de miellat et ne doivent donner qu'un premier point de repère.

\section{4: Auteurs}

\begin{tabular}{|c|c|c|c|}
\hline 1 & 2 & 3 & 4 \\
\hline & Coccina & & \\
\hline $\mathbf{F}$ & Physokermes hemicryphus Dalman & 35,9 & GONTARSKI 1940 \\
\hline $\mathbf{F}$ & Kermes quercus (Linnaeus) & 39,9 & » \\
\hline F & Trabutina mannipara (Ehrhorn) & 55 & Benthelot zit. in WeBER 1930 \\
\hline $\mathbf{F}$ & Coccus hesperidum Linnaeus & $\begin{array}{l}\text { sehr viel } \\
\text { grande quantité }\end{array}$ & WoLF und Ewart 1955 \\
\hline $\mathbf{F}$ & Icerya purchasi (Maskell) & $\begin{array}{l}\text { sehr viel } \\
\text { grande quantité }\end{array}$ & Ewart und Metcalf 1956 \\
\hline $\mathbf{F}$ & Planococcus citri (Risso) & $\begin{array}{l}\text { sehr viel } \\
\text { grande quantité }\end{array}$ & $\begin{array}{l}\text { EWart und Metcalf 1956, Gray } \\
\text { und Fraenkel 1954 }\end{array}$ \\
\hline $\mathbf{F}$ & Planococcus vitis Nied. & $\begin{array}{l}\text { viel, diskut. Fehlen } \\
\text { einer Darm-Inver- } \\
\text { tase } \\
\text { beaucoup, absence dis- } \\
\text { cutée d'une invertase } \\
\text { de l'intestin }\end{array}$ & Saleh und Salama 1971 \\
\hline $\mathbf{F}$ & Sacchariococcus sacchari (Cockerell) & " & Salama und Rizk 1969 \\
\hline $\mathrm{F}$ & Eulecanium coryli Linnaeus & $\begin{array}{l}\text { ansehnliche Menge } \\
\text { quantité importante }\end{array}$ & Bacon und Dickinson 1957 \\
\hline & Aphidina & & \\
\hline $\mathbf{F}$ & Cinara laricis (Hartig) & 47,3 & ARnhart 1927 \\
\hline $\mathbf{F}$ & Cinara pectinatae (Nördlinger) & 11,1 & GONTARSKI 1940 \\
\hline $\mathbf{F}$ & " & 25,3 & GeINITz 1930 \\
\hline $\mathbf{F}$ & Cinara piceae (Panzer) & 36,2 & GONTARSKI 1940 \\
\hline $\mathbf{F}$ & Lachnus roboris (Linnaeus) & 25,9 & Michel 1942 \\
\hline $\mathbf{F}$ & Tuberolachnus salignus (Gmelin) & $\begin{array}{l}\text { in erster Näherung } \\
\text { ca. } 25 \\
\text { en première approxi- } \\
\text { nation environ } 25\end{array}$ & MittLer 1958 \\
\hline
\end{tabular}




\begin{tabular}{|c|c|c|c|}
\hline I & 2 & 3 & 4 \\
\hline $\mathrm{F}$ & Cinara pilicornis (Hartig) & $\begin{array}{l}\text { sehr viel } \\
\text { grande quantité }\end{array}$ & MaURizio 1965 \\
\hline $\mathrm{F}$ & Cinara confinis (Koch) & 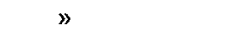 & $»$ \\
\hline $\mathbf{F}$ & Cinara pinea (Mordvilko) & 》 & » \\
\hline $\mathrm{F}$ & Cinara stroyani (Fasek) & $\begin{array}{l}\text { viel } \\
\text { beaucoup }\end{array}$ & » \\
\hline $\mathrm{F}$ & Cinara cuneomaculata (del Guercio) & $»$ & " \\
\hline F & Eucallipterus tiliae (Linnaeus) & 39 & Bacon und Dickinson 1957 \\
\hline F & $\begin{array}{l}\text { Drepanosiphum platanoides } \\
\text { (Schrank) }\end{array}$ & 55,4 & BüSGEN 1891 \\
\hline $\mathbf{F}$ & $\begin{array}{l}\text { Drepanosiphum platanoides } \\
\text { (Schrank) }\end{array}$ & $\begin{array}{l}\text { bedeutend } \\
\text { important }\end{array}$ & Bacon und Dickinson 1957 \\
\hline 0 & Schizoneura lanuginosa Hartig & 0,5 & Duspiva 1954 \\
\hline 0 & Megoura viciae (Buckton) & $<1$ & EHRHARDT 1962 \\
\hline 0 & Brevicoryne brassicae (Linnaeus) & $\begin{array}{l}\text { vereinzelt Spuren } \\
\text { traces isolées }\end{array}$ & Lамв 1959 \\
\hline 0 & Lipaphis erysimi (Kaltonbach) & $\begin{array}{l}\text { nichts gafunden } \\
\text { aucunes traces }\end{array}$ & Singh Sidhu und Patron 1970 \\
\hline $\mathbf{0}$ & Aulacorthum circumflexu. (Juckton) & 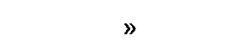 & Maltais und Auclair 1952 \\
\hline 0 & Macrosiphum rosae (Linnae is) & $\begin{array}{l}\text { wenig } \\
\text { peu }\end{array}$ & Duspiva 1954 \\
\hline 0 & Aphis pomi de Geer & $\begin{array}{l}\text { nichts gefunden } \\
\text { aucunes traces }\end{array}$ & $»$ \\
\hline 0 & Myzus persicae (Sulzer) & $\begin{array}{l}\text { seten Spuren } \\
\text { de rares traces }\end{array}$ & KUNKEL unpubl. \\
\hline (?) & $\begin{array}{l}\text { Thelaxes dryophila (Schrank) } \\
\text { Nach BöRNER (1938) } \\
\text { ohne Filterkammer } \\
\text { D'après Börnen (1938) pas de chambre } \\
\text { filtrante }\end{array}$ & $\begin{array}{l}\text { ? Ausnahme viel } \\
\text { ? exception beaucoup }\end{array}$ & MaURizio 1965 \\
\hline
\end{tabular}


s. unten) wandert zu 50-70\% den ( langen Weg » durch den Mitteldarm bzw. Hämolymphe, der Rest über die Filterkammer.

Spekuliert man über den Weg des Wassers, so könnte man die Kotfrequenzen bei Aphiden zu Hilfe nehmen. Filterdarmtiere haben nach Tabelle 1 eine 2-3 mal höhere Abgabefrequenz, was vermuten läßt, daß nur 33-50\% des Wassers den “ langen Weg » nimmt. Natürlich sind diese Zahlen bisher nur unsicher begründet, vor weiterreichenden Schlußfolgerungen sollte man sich hüten.

\section{3. - WEITERE KOHLENHYDRATE IM HONIGTAU}

\section{Fructose und Glucose}

Freie Fructose ist in vielen Honigtauen gegenüber der freien Glucose mengenmäßig stärker vertreten. So gibt z.B. ERнARDt (1962, Megoura viciae (Bucктоn)) 22 Gesamtkohlenhydrat-Prozente Fructose gegenüber $9 \%$ freier Glucose an, Bacon und Dickinson (1956, Eucallipterus tiliae (Linnaeus)) 14 gegenüber $4 \%$. Die Glucose liegt in Trehalose und höheren Transglukosidierungsprodukten (Oligosaccharide) gebunden vor, in Adelgiden-Honigtauen wohl ausschließlich (KUNKEL, unpubl.).

Bei der Mengenbestimmung von Fructose und Glucose insgesamt in freier und gebundener Form (Hertel und KunkeL, 1976, 1977, Larvenstadien von Myzus persicae (Sulzer) auf künstlicher Diät) ergab sich, daß Fructose nur ca. $17 \%$ des Gesamtkohlenhydrat-Gehaltes vertritt, obwohl sie aus der Diät (als Saccharose) zu $50 \%$ aufgenommen wird. Der Glucose-Wert schwankt auch viel extremer im Laufe der postembryonalen Entwicklung und repräsentiert wohl viel stärker den Ablauf von physiologischen Prozessen im Bereich Mitteldarm und Hämolymphe. Ehrharot (1962, M. viciae) fand übrigens eine Isomerasen-Aktivität im Darm. Leider ist über die Verdauungsphysiologie bei Insekten so überaus wenig bekannt (s. auch Hertel und KunKeI, 1976).

\section{Trehalose}

Der “ Blutzucker » der Insekten erweist sich als ein kontinuierlicher Bestandteil des Honigtaues. Ehrhardt (1962, M. viciae) fand immerhin einen $35 \%$ Anteil an den Gesamt-Kohlenhydraten. Ältere Autoren rechneten Trehalose unter " Dextrine ». Vielleicht ist Trehalose auch schon einmal als Maltose verbucht worden.

Trehalose kann durchaus als Substrat für Transglukosidierungs-Prozesse dienen (Ehrhardt, 1962). Ein Trisaccharid bestehend aus Trehalose, an der 
in bisher nicht bekannter Weise Glucose gebunden ist, bildet den Hauptanteil des Blutzuckers bei Adelgiden und Phylloxeriden (KunKel, unpubl.). Dementsprechend sind auch die Oligosaccharide im Honigtau der Adelgiden sehr komplex, z.T. nimmt auch noch Galactose daran teil, die in dem Nährgewebe der Ananasgallen frei vorliegt (Kunkel, 1966).

Bisher wurde von uns kein Honigtau ohne Trehalose gefunden. (z.B. Pseudococcus maritimus EнRH., diverse Adelgiden-Arten, Pemphigus bursarius (Linnaeus), Prociphilus xylostei (De Geer), Aphis craccivora Кoch, Aphis rumicis Linnaeus, Myzus persicae (Sulzer), Acyrthosiphon pisum (Harris)).

\section{Raffinose}

Die Verbreitung von Raffinose und $\alpha$-Galactosidasen : Heute weiß man, daß Raffinose (und evtl. weitere Glieder der Raffinose-Reihe) als ein die Saccharose begleitender Zucker (von 0 bis etwa $30 \%$ der Saccharose) im Phloem der weitaus meisten bisher untersuchten Pflanzen vorhanden ist. Eine gute Übersicht geben Zimmermann und Ziegler (1975). Dem entspricht auch eine weite Verbreitung von $\alpha$-Galactosidasen in den Darmtrakten von Cocciden und Aphiden (u.a. Duspiva, 1954, Ehrhardt, 1962, eigene Untersuchungen). Dabei dürften die Aktivitäten einer $\alpha$-Glucosidase zu der einer $\alpha$-Galactosidase in ähnlicher Relation stehen wie das Mengenverhältnis von Saccharose zu Raffinose in der Nahrung. Ein Hinweis auf eine ß- h-Fructosidase - das Erscheinen von Melibiose - konnte nur bei Cocciden erhalten werden.

Bei Honigtau-Analysen wird Raffinose nur selten angegeben. Oleaceae haben z.B. einen hohen Raffinose-Anteil, so daß es nicht überrascht, daß der Honigtau von Psyllopsis fraxinicola (Förster) ebenfalls viel Raffinose bzw. auch Glieder der Raffinose-Serie aufweist (Bacon und Dickinson, 1956). Daraus aber zu schließen, daß diese Psyllide Raffinose nicht verwerten kann, halten wir für voreilig. Wahrscheinlich werden Anteile von Raffinose über die Filterkammer schneller aus dem. Körper ausgeschleust. Übrigens fanden die Autoren sogar Melibiose im Honigtau, was man als Indiz für die Aktivität einer B- h-Fructosidase werten könnte. Bei einer Reihe von Cinara-Arten an Koniferen gibt Haragsim (1963) Raffinose an, was aber anscheinend von Maurizio (1966) nicht unterstützt werden kann. Ziegler und Mittler (1959) fanden in Phloemsaft von Picea abies keine, Chararas (1962) dagegen im Rindengewebe sehr wohl eine Raffinose. Salama und Rizk (1969); Saler und Salama (1971) geben den Zucker im Honigtau zweier Cocciden an. Wahrscheinlich dürfte Raffinose in vielen Honigtauen bisher noch nicht identifiziert worden sein.

Im Nektar ist Raffinose äußerst selten (LÜTtGe, 1962), so daß man durchaus diskutieren sollte, ob die $\alpha$-Galactosidase im Bienendarm eine Anpassung an die Honigtau-Tracht darstellt. 


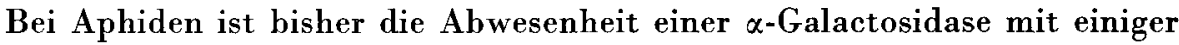
Sicherheit nur bei Megoura viciae (Bucktov) (Ehrhardt, 1962) und Acyrthosiphon pisum (Harris) (Srivastava und Auclair, 1962) bekannt. Dies konnten wir für beide Spezies bestätigen. Ausschließlich Wirtspflanzen beider Aphiden sind (krautige) Fabaceen, eine Gruppe, bei der nach ZimmerMANN und Ziegler (1975) nur äußerst selten und dann nur in Spuren Raffinose im Phloemsaft gefunden worden ist.

Vergleich von Myzus persicae und Acyrthosiphon pisum an einer künstlichen Raffinose-Diät

Exakte physiologische Einsichten lassen sich bei Aphiden eigentlich nur mit Hilfe einer Diät gewinnen. A. pisum (ohne $\alpha$-Galactosidase) und M. persicae (mit einer $\alpha$-Galactosidase) ist auf der gleichen künstlichen Diät zu halten (nach Mittler, 1966, modifizierte Diät, Kunkel, 1977 : diet a 3). Die $15 \%$ Saccharose der Kontroll-Diät (1) wurde durch $10 \%$ Saccharose $+5 \%$ Raffinose (2), und durch $10 \%$ Raffinose (3) ersetzt (Kunkel, unpubl.). Solche hohen Raffinose-Werte kommen in der Natur als Phloemsaft-Anteil sicher nicht vor. Bei diesem Vorversuch sollten aber Tendenzen abgeschätzt werden.

Mit anderen Diät-Formulationen und z.T. auch anderem Erfolg haben schon Auclair (1967, Aphis gossypii Glover) und Mittler et al. (1970, Myzus persicae (SulZER)) Raffinose angeboten. Gegenüber letzteren Autoren lagen bei unseren Versuchen die Überlebensquoten und Reproduktionsraten günstiger.

Doch das Reizvolle unseres Vorversuches war die Gegenüberstellung beider ernährungsphysiologisch unterschiedlichen Aphiden-Spezies. Wie erwartet, zeigen die Ergebnisse - Überlebensdauer der Mütter in Tabelle 4, Reproduktionsrate in Tabelle 5 - tatsächlich große Unterschiede. Die Differenz in Tabelle 5 würde noch deutlicher ins Auge fallen, wenn die jeweiligen LarvenAnzahlen nicht auf die gerade noch lebenden Mütter, sondern auf deren Ausgangszahl bezogen wären.

Auch für $M$. persicae erscheint eine Diät mit solch einem hohen RaffinoseAnteil unphysiologisch. Die Zahl der lebenden Mütter sinkt gegenüber dem Standard (Tabelle 3), ebenfalls die Reproduktionsrate. Die reine RaffinoseDiät wird vielleicht aus geschmacklichen Gründen am ersten Tag nur zögernd angenommen (Reproduktionsrate 1,57, s. KUNKEL, 1977).

\section{4. - MYZUS PERSICAE AUF KÜNSTLICHER DIÄT, EIN MODELL}

Honigtau der Pfirsichblattlaus $M$. persicae wird gelegentlich von Bienen gesammelt (SChMutTERER, 1953, 1958). 
TAB. 4. - Am Leben gebliebene Mütter in \% der jeweils in der Kontrolle (15\% Saccharose-Diät) noch lebenden Individuen (=100\%).

Ausgangszahlen : $M$. persicae jeweils $(3 \times) 60, A$. pisum jeweils $(3 \times)$ 30 Individuen. (2) Diät mit $10 \%$ Sacch. und $5 \%$ Raff., (3) $10 \%$ Raff.

TABL. 4. - Pourcentages de femelles restées en vie par rapport aux têmoins encore vivants $(=100 \%$ ) sur régime à $15 \%$ de saccharose.

$M$. persicae: 3 lots de 60 individus chacun

A. pisum : 3 lots de 30 individus chacun

(2) : régime comprenant $10 \%$ de saccharose et $5 \%$ de raffinose

(3) : régime comprenant $10 \%$ de raffinose

\begin{tabular}{|c|c|c|c|c|c|c|}
\hline & 1. & 2. & 3. & 4. & 5. & 6. Tag \\
\hline \multicolumn{7}{|l|}{ (2) } \\
\hline M. persicae & 100 & 100 & 100 & 96,4 & 92,9 & 85,7 \\
\hline A. pisum $\ldots \ldots \ldots$ & 100 & 100 & 73,3 & 33,3 & 13,3 & 6,7 \\
\hline \multicolumn{7}{|l|}{ (3) } \\
\hline$M \cdot$ persicae & 100 & 100 & 100 & 96,8 & 96,8 & 90,3 \\
\hline A. pisum ....... & 100 & 93,3 & 60,0 & 20,0 & 0,0 & 0,0 \\
\hline
\end{tabular}

TAB. 5. - Durchschnittliche Reproduktionsrate (abgegebene Larven pro lebender Mutter und 24 Std) im Vergleich mit den verschiedenen Diäten : (1) $15 \%$ Sacch., (2) $10 \%$ Sacch. $+5 \%$ Raff., (3) $10 \%$ Raff.

TABL. 5. - Taux moyen de reproduction (larves nées en 24. $h$ par femelle vivante) selon les différents régimes :
(1) : $15 \%$ de saccharose
(2) : $10 \%$ de saccharose $+5 \%$ de raffinose
(3) : $10 \%$ de raffinose

M. persicae (jeweils 60 Mütter zu Beginn der Versuche) (lots de 60 femelles en début d'expérience)

\begin{tabular}{|c|c|c|c|c|c|c|}
\hline & $\begin{array}{l}\text { 1. Tag } \\
1^{\text {jour }}\end{array}$ & $\begin{array}{l}\text { 2. Tag } \\
\text { 2e jour }\end{array}$ & $\begin{array}{l}\text { 3. Tag } \\
\text { 3e jour }^{2}\end{array}$ & $\begin{array}{l}\text { 4. Tag } \\
\text { 4e jour }\end{array}$ & $\begin{array}{l}\text { 5. Tag } \\
5^{\mathrm{e}} \text { jour }\end{array}$ & $\begin{array}{l}\text { 6. Tag } \\
6^{\mathrm{e}} \text { jour }\end{array}$ \\
\hline (1) & $4,4,7$ & 6,61 & 5,22 & 4,80 & 4,95 & 3,52 \\
\hline (2) & 6,42 & 6,11 & 3,95 & 4,56 & 3,47 & 0,33 \\
\hline (3) & 1,57 & 5,05 & 3,92 & 3,67 & 3,26 & 2,00 \\
\hline
\end{tabular}

A. pisum (jeweils 30 Mütter zu Beginn der Versuche) (lots de 30 femelles en début d'expérience)

\begin{tabular}{|c|c|c|c|c|c|c|}
\hline & $\begin{array}{l}\text { 1. Tag } \\
\text { ler jour }^{2}\end{array}$ & $\begin{array}{l}\text { 2. Tag } \\
2 \mathrm{e} \text { jour }\end{array}$ & $\begin{array}{l}\text { 3. Tag! } \\
3^{\ominus} \text { jour }\end{array}$ & $\begin{array}{l}\text { 4. Tag } \\
4 \text { ej our }\end{array}$ & $\begin{array}{c}5 . \text { Tag } \\
5 \text { e jour }\end{array}$ & $\begin{array}{l}\text { 6. Tag } \\
\text { 6e jour }\end{array}$ \\
\hline (1) & 4,20 & 8,22 & 6,56 & 6,40 & 5,08 & 5,23 \\
\hline (2) & 2,36 & 6,58 & 1,58 & 2,30 & 0,80 & 0,80 \\
\hline (3) & 2,25 & 2,11 & 0,55 & 0,90 & 0,00 & 0,00 \\
\hline
\end{tabular}


Da für diese Blattlaus eine recht gute künstliche Diät entwickelt worden ist (Ủbersicht : KunkEL, 1977), ergab sich zum ersten Male die Möglichkeit, Nahrungsaufnahme-Menge, Körpergewichtszunahme, Transpiration, Respiration und Exkretion direkt zu messen. Der Fortschritt einer Trennung von der sich ständig verändernden Wirtspflanze mit der unbekannten jeweiligen Zusammensetzung des Phloem-Saftes wird erkauft durch künstliche Bedingungen an der Diät. Doch passen die erzielten Ergebnisse (Honigtau-Abgabefrequenz, Lebendgewicht, Reproduktionsrate, Kotzusammensetzung, Ausnutzung der Nahrung im Hinblick auf den Stickstoff-Haushalt etc.) durchaus in den Rahmen dessen, was von der Pflanze her bekannt ist.

Für das dritte Larvenstadium konnten bereits Stoffmengen, Aminosäure (N)-, Glucose-, Wasser- und Energie-Bilanzen vorgelegt werden (K UNKEL und Hertel, 1975). Für den kurzen Zeitabschnitt zu Beginn des 4. Stadiums wurde die Nahrungsaufnahme, Frischgewichtszunahme des Körpers, der Verlauf der Gesamtkohlenhydrat-und Stickstoff-Abgabe als Honigtau miteinander in Beziehung gesetzt (Hertel und KunKel, 1976). Besonders faszinierend ist, daß bei diesen Versuchen die Zusammensetzung der Nahrung bekannt und konstant war.

Ein wichtiges Ergebnis erscheint uns, daß die geflügelten und ungeflügelten Morphen auch verschiedene ernährungs-physiologische Typen repräsentieren. Dies zeigt sich besonders in der Relation von Kohlenhydrat- und Stickstoff-Bilanzen. Grundsätzlich nutzen beide Morphen aber nicht nur die Aminosäuren aus, wie es häufig vereinfachend angenommen wird. Die als Pflanzenparasiten stärker spezialisierten apteren Morphen mit ihrer höheren Reproduktion nutzen die Aminosäuren zu $70 \%$ aus, die alaten zu 64 \%; die Kohlenhydrate dagegen entsprechend zu $30 \%$ bzw. $58 \%$ : der jeweilige Rest wird als Honigtau ausgeschieden. Die Alaten bauen mit den Kohlenhydraten ihre etwa $50 \%$ höheren Fettreserven auf, die sie für den Ausbreitungsflug brauchen.

Gerade im Hinblick auf Honigtau-Probleme wäre eine ähnliche Untersuchung an einer Blatt- oder Schildlaus mit Filterkammer sehr interessant. Die Honigtaue beider Typen (mit und ohne Filterkammer) erscheinen im Hinblick auf die Relation Stickstoff zu Kohlenhydraten nicht sehr unterschiedlich (Michel, 1942, Lindemann, 1948, Maltais und Auclair, 1952, Mitéler, 1953, Ehrhardt, 1962 etc.). Uns sind nur zwei Fälle bekannt, bei denen zugleich direkt Nahrung und Honigtau im Hinblick auf dieses Problem verglichen werden kann : Ziegler, 1956, Coccide an Efeu (? Coccus hesperidum Linnaeus, ? Stadium), und Ehrhardt, 1962, M. viciae, aptere Adulte.

In allererster Näherung könnte man die Gewichte von Kohlenhydraten (angenommen : $1 \mathrm{~g}$ ) und Aminosäuren (x g, Leucin-Äquivalente) in Beziehung 
setzen. Betrachtet man dann die x g Aminosäuren der Nahrung als $100 \%$, erhält man die Werte für Honigtau in der Tabelle 6.

TAB. 6. - Unterschiedliche Aminosäuren-Resorption bei zwei Aphiden (ohne Filterdarm) und einer Coccide (mit Filterdarm). Der Gewichtsanteil Aminosäuren (Leucin-Äquivalente) im Verhältnis zu einer Gewichtseinheit Kohlenhydrat (Glucose-Äquivalent) wird für die Nahrung jeweils mit $100 \%$ angenommen. Falls Kohlenhydrate und Aminosäuren in gleicher Relation zueinander resorbiert werden, ergeben sich für den Gewichtsanteil Aminosäuren im Honigtau wiederum diese $100 \%$. Stattdessen wurden folgende «Werte (\%) Honigtau » gefunden.

TABL. 6. - Différence de résorption des acides aminés chez 2 Aphides (sans chambre filtrante) et un Coccide (possêdant une chambre filtrante). On attribue à la nourriture un rapport en poids de $100 \%$ des acides aminés (équivalents en leucine) à une unité de poids d'hydrate de carbone (équivalent en glucose). Dans le cas où les hydrates de carbone et les acides aminés sont résorbés dans la même proportion, le rapport en poids des acides aminés au miellat est également de $100 \%$. Dans le cas contraire on a trouvé les valeurs suivantes en « $\%$ de miellat ».

\begin{tabular}{|c|c|c|c|}
\hline $\begin{array}{l}\text { Spezies } \\
\text { Espèce }\end{array}$ & $\begin{array}{c}\text { Nahrungsquelle } \\
\text { Source } \\
\text { de nourriture }\end{array}$ & $\begin{array}{l}\text { Werte }(\%) \\
\text { Honigtau } \\
\% \text { miellat }\end{array}$ & $\begin{array}{l}\text { Autoren } \\
\text { Auteurs }\end{array}$ \\
\hline $\begin{array}{c}M . \text { persicae } \\
\text { alat } \mathrm{L} 3 \\
\text { apter } \mathrm{L} 3\end{array}$ & Diät & $\begin{array}{l}87,3 \\
40,9\end{array}$ & $\begin{array}{l}\text { KUNKEL und HERTEL } 1975 \\
\text { (Durchschnittswerte) }\end{array}$ \\
\hline $\begin{array}{l}\text { M. vicae } \\
\text { aptere Adulte }\end{array}$ & Vicia & 52,7 & ЕнRHARDT 1962 \\
\hline $\begin{array}{l}\text { ? C. hesperidium } \\
\text { ? Stadium }\end{array}$ & Hedera & 0,7 & ZIEGLER 1956 \\
\hline
\end{tabular}

Die Coccide mit dem komplizierten Filterdarm hat offenbar insgesamt gesehen einen extrem hohen Anteil an Aminosäuren in den Körper aufgenommen. Bei diesen Zahlen sollte man bedenken, daß bei der Verwertung es auf die jeweiligen absoluten Konzentrationen beider Komponenten in der Nahrung ankommt (Hertel und KunKel, 1977). Die Bezugsgröße Kohlenhydrat ist weiterhin keine Konstante. So verwenden die alaten Morphen von $M$. persicae Kohlenhydrate für die Bereitung von Reservestoffen.

Selbstverständlich muß man bei allen Honigtau-Analysen wissen, daß eine hohe Anzahl variabler Faktoren auf dessen Zusammensetzung einwirkt.

Hertel und Kunkel (1976) hielten Nahrung und abiotische Faktoren möglichst konstant, als sie die erste Hälfte des 4. Larvenstadiums (apter) untersuchten. Die Zusammensetzung des Honigtaues wechselte ständig. Zuerst wurde der Nahrung vor allem Wasser entnommen (um sich damit zum neuen Körpervolumen auszudehnen). Bis zur Stadienmitte wurden besonders große Mengen Aminosäuren entnommen, nachfolgend mehr Kohlenhydrate. 
Auf eine Reduktion sämtlicher Aminosäuren um $50 \%$ reagierten die Larven je nach ontogenetischem Alter unterschiedlich. Eine Hyperphagie (bis zu $20 \%$ gesteigerte Nahrungsaufnahme) trat nur ab Stadienmitte auf. Grundsätzlich aber wurde der Ausgleich durch Reduktion (um ca. $35 \%$ ) der Wachstumsrate gefunden : Es entstehen die auch in der Natur bekannten sogn. Minus-Apteren (KunKel und KLoft 1974). Diese Tiere steigern überraschenderweise nicht ihre Nahrungsaufnahme.

Die filterdarmlosen $M$. persicae konnten aus der um $50 \%$ an Aminosäuren ärmeren Diät nicht einmal proportional einen (gegenüber Honigtau an der Standardlösung $=100 \%$ ) um $50 \%$ herabgesetzten Anteil entnehmen, allerdings immerhin 45,8\% (HerteL und KunkeL, 1977). Bei gleicher Nahrungsaufnahme-Menge und einer Reduktion um $35 \%$ an Körpergewichtszunahme scheiden sie erwartungsgemäß bedeutend mehr Honigtau aus (über $40 \% \mathrm{mehr}$, KuNKEL und HerteL, 1976).

Nach Erhöhung des Aminosäure-Anteils (150\% und $200 \%$ ) verringert sich dessen Resorption exponentiell (Hertel und Kunkel, 1977). Dabei nähert sich das Verhältnis Stickstoff zu Kohlendydrate im Honigtau etwas dem des Nahrungsangebotes, wobei allerdings die ungefähre Relation 10 : 1 beider Quotienten gewahrt bleibt. Offenbar ist in der Nähe der StandardDiät-Konzentration eine optimale Ausnutzung des Nahrungssaftes im Hinblick auf die Aminosäure-Resorption erreicht. Bei geringerer Konzentration läßt sich die Aufnahme nicht steigern, für höhere Konzentrationen scheint der Darm oder der Gesamtorganismus ebenfalls nicht entsprechend gerüstet zu sein. Auffallenderweise liegt das Stickstoff/Kohlenhydrat-Verhältnis im Honigtau von Läusen an ihrer Wirtspflanze im gleichen Bereich; und das Verhältnis in der Standard-Diät wurde experimentell erarbeitet (u.a. optimale Körpergewichtszunahme der Tiere).

\section{5. - BEZIEHUNGEN VON APHIDEN ZUR WIRTSPFLANZE}

$\mathrm{Zu}$ diesem Thema seien abschließend einige kurze Bemerkungen gemacht.

\section{Jahreszeitliche Abhängigkeiten}

Für die Honigtau-Tracht ist es von Bedeutung, daß die Populationen von Schild- und Blattläusen eine sommerliche Depression erleiden, im Herbst sich aber bei manchen Aphiden wieder aufbauen. Von dieser Regel gibt es Abweichungen (bei Gallbewohnern verlängert sich die Frühjahrs-Saison, Kunkel, 1966; krautige Pflanzen haben oft andere Vegetations-Zeiten; Lokalbibitoren sind manchmal nicht betroffen, KuNKeL, 1967). 
Seit Lindemann (1948) wird der sinkende Gehalt an Aminosäuren im Phloemsaft dafür verantwortlich gemacht (s. auch Ziegcer, 1956). Doch auch andere Substanzen erreichen im Sommer eine um 50-80\% niedrigere Konzentration, um im Herbst wieder anzusteigen.

Bisher bekannt dafür sind myo-Inosit (KLuge, 1970), AdenosinTriphosphat (Becker, 1973; Ziegler, 1975) und Kalium (Dixon, 1975, indirekter Hinweis durch Honigtau-Analysen).

Aufgrund von Versuchen mit künstlichen Diäten wissen wir, daß besonders der Level an Aminosäuren und Kalium Wachstum und Reproduktion von Aphiden bestimmen, doch auch myo-Inosit und ATP wesentlichen Einfluß nehmen.

Interessanterweise können die Blattläuse gerade diese Stoffe aus der komplexen Diät herausschmecken, wie Wahlversuche zeigen (Übersicht Kunkel, 1977). Außer sehr wenigen Aminosäuren (vor allem Methionin) werden einzelne Aminosäuren geschmacklich nicht diskriminiert, ebenfalls keine Vitamine (bei Ascorbinsäure aber u.U. kurzzeitig eine Ablehnung) und die meisten anorganischen Ionen.

\section{Vergleich Phloemsaft-Diät}

Je länger mit einer künstlichen Diät gearbeitet wird, desto unbekannter erscheint der Phloemsaft, dessen Kopie man doch anstrebt. Willkürlich seien einige auftauchende Fragen erwähnt (s. auch KunKel, 1977). Blattläuse arrangieren sich mit einer Diät ohne Überdruck, müssen sie es auch manchmal an ihrer Wirtspflanze? Sie bevorzugen einen leicht alkalischen $\mathrm{pH}$, den sie auch in ihrer Wirtspflanze finden, während in diesem Bereich in der künstlichen Diät Metallphosphate und -hydroxide präzipitieren. Falls Sterole im Phloemsaft vorkommen - Forrest und KnIghts (1972, M. persicae) bringen durch ihre Honigtau-Analysen ein indirektes Indiz; Aкеу und Beck (1972, A. pisum) konnten durch Hinzufügen von Cholesterin eine Wachstumsförderung erzielen; Ehrhardt (1968, Aulacorthum circumflexum) wies eine ausreichende Synthese von Cholesterin allein durch Blattlaus-Symbionten nach - : wie werden diese hydrophoben Substanzen dispergiert (Träger oder Netzmittel ?). Welche Substanzen fungieren als Chelatbildner für Spurenelemente (Ascorbinsäure, Zitronensäure ?), welche als Antioxidantien ? Welche Substanzen sind noch im Phloemsaft zu erwarten? Haben Pflanzenhormone oder Zuckerphosphate eine Bedeutung für die Blattläuse? Freie RNA scheint im Phloemsaft vorzukommen (Ziegler, 1975), und auch in der Diät förderlich zu sein (DADD, 1967). Unseres Wissens haben Botaniker Folsäure im Phloem noch nicht eindeutig nachgewiesen, alle bisher untersuchten Aphiden sind aber auf eine Zufuhr streng angewiesen. Riboflavin dagegen müßte im Aphiden- 
körper synthetisiert werden, denn es ist bisher im Phloemsaft nicht sicher nachgewiesen, wirkt in der Diät als Deterrent und steht im Verdacht, als Chelat-Bildner die Spurenelemente zu fest zu halten.

\section{Einflu $\beta$ auf die Wirtspflanze}

Die Vorstellung, daß durch Speicheln (KLOFT, 1960, Lamb et al. 1967) in die Pflanze diese zugunsten der Ernährung von Schild- und Blattläusen verändert wird, ist schon vor einiger Zeit in unserem Hause sehr gepflegt worden (KцоFт 1950, 1956, 1956/60, 1960, KunkeL, 1966, 1967, 1968 a, 1968 b). Um die enge Beziehung zu den morphologisch definierten Gallen zu unterstreichen, wurde der Begriff “ Physiologische Galle 》 eingeführt (KLofT, 1956/1960). In der Zwischenzeit konnte durch elegante Versuche (Besiedlung des gleichen Blattareals jeweils sowohl von der Ober- als auch von der Unterseite, Besiedlung mit einem oder mehreren Individuen) diese Vorstellung untermauert werden (WaY und Cammele, 1970; Dixon und Wratten, 1971; SheARER, 1976).

Jene Ergebnisse haben natürlich Einfluß auf Überlegungen, wie nützlich Honigtau-Erzeuger sein können; oder inwieweit durch Experimente mit Blattläusen von den Botanikern ein " echtes » Bild der Phloemverhältnisse erhalten wird.

Der Honigtau dürfte nach all dem, was oben vorgebracht worden ist, sehr komplex und sehr variable zusammengesetzt sein. Es fehlen Untersuchungen, ob Bienen ihn nur als Zuckerlösung verwenden oder ob er ihnen mehr bietet.

Eingegangen im Mai 1977.

Reçu pour publication en mai 1977.

\section{RESUMÉ}

En Europe centrale les abeilles ne récoltent le miellat que sur peu d'espèces (58 connues) de Sternorrhynches (Tabl. 1). Cette récolte est favorisée près des fleurs par des accumulations, dans les galles et dans les branches périphériques par une moindre visite des fourmis. Mais $72,4 \%$ de toutes les espèces possèdent une chambre filtrante. En conséquence ces pucerons sécrètent 2 à 3 fois plus de miellat et ce miellat renferme $25-40 \%$ de saccharose en poids sec au lieu de $1 \%$ chez les pucerons qui n'ont pas de chambre filtrante. On discute de la présence d'autres hydrates de carbone. Le tréhalose semble présent dans tous les miellats. Puisque le raffinose est connu pour être largement répandu dans la sève de phloème, sa présence dans le miellat est discutée. Tous les pucerons ne peuvent pas assimiler le raffinose, comme le prouvent les études sur milieux artificiels. Le miellat de $M$. persicae est récolté à l'occasion par les abeilles. Puisque, par ailleurs, cette espèce s'élève bien sur milieu artificiel, on a pu étudier intensivement la physiologie de la nutrition. On fait part de certains de ces résultats. A la fin on montre l'importance des plantes-hôtes dans les questions suivantes : dépression estivale des populations de pucerons, analyse de la sève de phloème, influence de la salive dans la nutrition. 


\section{LITERATUR}

Akey D. H., Beck S. D., 1972. - Nutrition of the pea aphid, Acyrthosiphon pisum : Requirement, for trace metals, sulphur and cholesterol, J. Insect Physiol., 18, 1901-1914.

Arvhart L., 1927. - Österreichischer Lärchenhonigtau, Lärchenmanna und Lärchenhonig. Z. angew. Entomol, 12, 457-472.

Auclair, J. L., 1959. - Feeding and exeretion by the pea aphid, Acyrthosiphon pisum (HARr.) (Homoptera : Aphididae) reared on different varieties of peas. Ent. exp. appl., 2, 279-286.

AUCLAIr J. L., 1967. - Effects of light and sugars on rearing the cotton aphid, Aphis gossypii, on a germ-free and holidic diet. J. Insect Physiol., 13, 1247-1268.

BACon J. S., Dickinson B., 1957. - The origin of melizitose : a biochemical relationship between the lime tree (Tilia spp.) and an aphid (Eucallipterus tiliae L.). Biochem. J., 66, 289-297.

Banks C. J., Nixon H. L., 1959. - The feeding and excretion rates of Aphis fabae Scop. on Vicia faba L. Ent. exp. appl., 2, 77-81.

Becker D., 1973. - Beiträge zur Kenntnis der biochemischen Leistung pflanzlicher Assimilatbahnen. Dissertation TU München, $102 \mathrm{p}$.

Berlese A., 1893. - Le Cocciniglie Italiane vivent : sugli agrumi. Rivista di Patologia vegetale, $2,129-193$.

BörNer C., 1938. - Neuer Beitrag zur Systematik und Stammesgeschichte der Blattläuse. Abh. Nat. Ver., Bremen, 30, 167-179.

Börner C., 1952. - Europae centralis Aphides. Die Blattläuse Mitteleuropas. Mitteilungen aier Thüringischen Bot. Gesellschaft Beiheft, 3, 488 p.

Büsger M., 1891. - Der Honigtau. Biologische Studien an Pflanzen und Pflanzenläusen. Jenaische Zeitschrift für Naturwissenschaft, 25, (NIF. 18) 333-428 und 2 Tafeln.

Chararas C., 1962. - Relations entre les variations de la pression osmotique des conifères et l'extension des Coleopteres Scolytidae. XI. Int. Kongr. Entomol. Wien 1960, 2, 246-249.

DADD R. H., 1967. - Improvement of synthetic diet for the aphid, Myzus persicae using plant juices, nucleid acids, or trace metals. J. Insect Physiol., 13, 763-778.

Drxon A. F. G., 1971. - The life-cycle and host preferences of the bird cherry-oat aphid, Rhopalosiphon padi L., and their bearing on the theories of host alternation in aphids. Ann. appl. Biol., 68, 135-147.

Dixon A. F. G., 1975. - Aphids and translocation, 154-170, in : M. H. Zimmermann, J. A. MilbuRN, eds., Transport in Plants I. Encyclopedia of Plant Physiology Vol. I. Springer-Verlag, Berlin, Heidelberg, New York, 535 p.

Dusprya F., 1954. - Enzymatische Prozesse bei der Honigtaubildung der Aphiden. Verh. Dtsch. Zool. Ges., (Tübingen), 440-447.

EhrHaRdT P., 1962. - Untersuchungen zur Stoffwechselphysiologie von Megoura viciae Buckt., einer phloemsaugenden Aphide. Z. vgl. Physiol., 46, 169-211.

Ehrhardt P., 1963. - Untersuchungen über Bau und Funktion des Verdauungstraktes von Megoura viciae Buckt. (Aphididae, Homoptera) unter besonderer Berücksichtigung der Nahrungsaufnahme und der Honigtauabgabe. Z. Morph. Ökol. Tiere, 52, 597-677.

Ehrhardt P., 1968. - Nachweis einer durch symbiontische Mikroorganismen bewirkten Sterinsynthese in künstlich ernährten Aphiden (Homoptera, Rhynchota, Insecta). Experientia, 24, 82.

Ewart W. H., Metcalf R. L., 1956. - Preliminary studies of sugars and aminoacids in the honeydew of five species of coccids feeding on Citrus in California. Ann. Ent. Soc. Am., 49, 441 .

Forrest J. M. S., Knights B. A., 1972. - Presence of phytosterols in the food of the aphid, Myzus persicae. J. Insect Physiol., 18, 723-728.

Fossel A., 1956. — Steirische Honige. Bienenvater, 77, 156-163.

Fossel A., 1963. - Aus dem Leben der europäischen Ahornblattlaus (Periphyllus villosus Htg.). Z. Bienenforsch., 6, 199-207. 
Fossel A., 1970. - Anleitung zur Determination einiger in Mitteleuropa verbreiteten Vertreter des Genus Cinara Curt. (Aphidoidae, Lachnidae). Waldhygiene, 8, 129-190.

Fosser A., 1971 a. - Neue Beobachtungen an Cinara cembrae (SEIrner, 1936) (Homoptera, Lachnidae). Ann. Zool., (Warsaw), 28, 353-364.

Fosser A., 1971 b. - Ist Cinara neubergi (ARnhard, 1930) eine selbständige Art? (Homoptera, Lachnidae). Ann. Zool., (Warsaw), 29, 1-14.

Fossel A., 1972 a. - Vergleichende Messungen an Cinara (C.) pinea (Mordv.) (Homoptera, Aphidina). Mitt. naturwiss. Ver. Steiermark, 102, 125-144.

Fossel A., 1972 b. - Die Variabilität von Cinara (C.) pini (L.) (Homoptera, Aphidina). Mitt. naturwiss. Ver. Steiermark, 102, 145-156.

Fossed A., 1972 c. - Die Populationsdichte einiger Honigtauerzeuger und ihre Abhängigkeit von der Betreuung durch Ameisen. Waldhygiene, 9, 185-191.

Geinitz B. C., 1930. - Die Entstehung des Tannenhonigs, Arch.f. Bienenkunde, 9, 308.

Gontarski H., 1940. - Beitrag zur Honigtaufrage. Z. angew. Entomol., 27, 321-332.

Gray H. E., Fraenkel G., 1954. - Fructomaltose a recently discovered trisaccharide isolated from honeydew. Science, 118, 304.

Haragsim O., 1963. - Honigtau und seine Ausnützung in der Imkerei. Wiss. Arb. d. Forschungsanstalt $f$. Bienenzucht in Dol., 3, 227.

Hertel R., Kunkel H., 1976. - Wechselnde Verwertung chemisch definierter Nahrung durch Myzus persicae-Larven (Hemiptera : Aphididae). Zool. Jb. Physiol., 80, 259-266.

Hertel R., Kunkel H., 1977. - Einige Faktoren, welche die Honigtauzusammensetzung natürlich wie auch holidisch ernährter Aphidenlarven beeinflussen. Apidologie 8, (4.), $427-436$.

Herzıg J., 1938. -- Ameisen und Blattläuse (Ein Beitrag zur Ökologie aphidophiler Ameisen). Z. angew. Entomol., 24, 367-437.

KLoft W., 1950. - Vergleichende Untersuchungen an einigen Cocciden und Aphiden. Verh. Dtsch. Zool. Ges. (Marburg), 229-296.

Kloft W., 1956. - Beeinflussung der Photosynthese und Atmung von Pflanzengeweben durch Pflanzenläuse. Naturwiss., 43, 65-66.

KlOFT W., 1956/1960. - Wechselwirkungen zwischen pflanzensaugenden Insekten und den von ihnen besogenen Pflanzengeweben. Habilitationsschrift Würzburg 1956. Z. angew. Entomol., 45, 337-381 und 46, 42-70.

KLOFT W., 1966. - Liste der bienenwirtzchaftlich wichtigen Honigtauerzeuger mitteleuropäischer Waldgebiete. Z. Bienenforschung, 8, 191-194.

KLUGE H., 1970. - Jahreszeitliche Schwankungen des Kohlenhydratgehaltes in Siebröhrensäften, Blättern und Wurzeln einiger Holzgewächse. Biochemie, Physiol. Pflanz., 161, 142-165.

KunKe H., 1966. - Ernährungsphysiologische Beziehungen der Sternorrhynchen zur Wirtspflanze unter besonderer Berïcksichtigung der Coccina und Aphidina. Dissertation Universität Bonn, 172 p. + Anhang.

Kunkel H., 1967. - Zur Ernährungsweise zweier Phylloxera-Arten (Phylloxeridae, Aphidina) an Eichenblättern. Z. angew. Zool., 54, 517-525.

KunKel H., 1968 a. - Untersuchungen über die Buchenwollschildlaus Cryptococcus fagi Bär. (Insecta, Coccina), einen Vertreter der Rindenparenchymsauger. Z. angew. Entomol., 61, 373-380.

Kunkel H., 1968 b. - Die Verteilung von ${ }^{32}$ P-markiertem Phosphat in Ulmus-Blättern unter Einfluß von Tetraneura ulmi (L.) (Rhynchota, Aphidina). Phytopath. Z., 62, 383-389.

Kunkel H., 1973. - Die Kotabgabe der Aphiden (Aphidina, Hemiptera) unter Einfluß von Ameisen. Bonn. Zool. Beitr., 24, 105-121.

KunKel H., 1977. - Membrane feeding systems in aphid research. In : Harris K. F., Maramorosci K. eds., Aphids as virus vectors. Academic Press London and New York. 311-338.

Kunkel H., Hertel R., 1975. - Erstellung einer Stoffwechselbilanz für beide virginoparen Morphen von Myzus persicae (Sutz.)-Larven im dritten Stadium. Feologia (Berl.), 18, 23-35. 
Kunkel H., Hertel R., 1976. - Kotabgabe von holidisch ernährten Myzus persicae-Larven (Aphidina, Homoptera) bei unterschiedlichen Experimentalbedingungen. Ent. exp. appl., 19, 82-95.

Kunkel H., Kloft W., 1974. - Polymorphismus bei Blattläusen, 152-201, in : Schmidt G. H. ed., Sozialpolymorphismus bei Insekten. Wissenschaftliche Verlagsgesellschaft mbH., Stuttgart, $974 \mathrm{p}$.

Laмв K. P., 1959. - Composition of the honeydew of the aphid Brevicoryne brassicae (L.) feeding on swedes (Brassica napobrassica DC.). J. Ins. Physiol., 3, 1-13.

Lamb K. P., Eнrhandt P., Moknicke V., 1967. - Labeling of aphid saliva with rubidium-86. Nature, 214, 602-603.

LeonhardT H., 1940. - Beiträge zur Kenntnis der Lachniden der wichtigsten Tannenhonigtauerzeuger. Z. angew. Entomol. 27, 208-272.

Lindemann Ch., 1948. - Beitrag zur Ernährungsphysiologie der Blattläuse. -- Z. vergl. Physiol. 31, 112-133.

Lürtge U., 1962. - U̇ber die Zusammensetzung des Nektars und den Mechanismus seiner Sekretion. III. Die Rolle der Rückresorption und der spezifischen Zuckersekretion. Planta, 59, 175-194.

Maltais J. B., Auclarr J. L., 1952. - Occurrence of amino acids in the honeydew of the crescent-marke lily aphid, Myzus circumflexus (Buck.). Canad. J. Zool., 30, 191-193.

Maurizio A., 1965. - Honigtau-Honigtauhonig, 157-185, in : Kooft W., Maurizio A., KaEser W. eds. Das Waldhonigbuch. Imkerfreund Bücher Bd. 3. Ehrenwirth Verlag München, 218 P.

Maurizio A., Grafl I., 1969. - Das Trachtpflanzenbuch. Imkerfreund Bücher, Bd. 4. Ehrenwirth Verlag München, $288 \mathrm{p}$.

Maxwell F. G., Painter R. H., 1959. - Factors affecting rate of honeydew deposition by Therioaphis maculata (Buck.) and Toxoptera graminum (Rond.). J. Econ. Entomol., 52, 368-373.

Michel E., 1942. - Beiträge zur Kenntnis von Lachnus (Pterochlorus) roboris L., einer wichtigen Honigtauerzeugerin an der Eiche. Z. angew. Entomol., 29, 243-281.

Mittler T. E., 1953. - Amino-acids in phloemsap and their excretion by aphids. Nature, 172, 207.

MitTler T. F., 1958. - The excretion of honey-dew by Tuberolachnus salignus (GMeLIN) (Homoptera, Aphididae). Proc. R. Entomol. Lond., 33, 49-55.

Mittcer T. E., 1970. - Uptake rates of plant sap and synthetic diet by the aphid Myzus persicae. Ann. Entomol. Soc. Am., 63, $1701-1705$.

Mittler T. E., Sylvester 1961. - A comparison of the injury to alfalfa by the aphids, Therioaphis maculata and Macrosiphum pisi. J. Econ. Entomol., 54, 615-622.

Mitteer T. E., Dadd R. H., Daniels S. C., 1970. - Utilization of different sugars by the aphid Myzus persicae. J. Insect Physiol., 16, 1873-1890.

Molliano M. C., 1931. - Observations sur les miellées produites par des pucerons. Rev. gen. botan., 43, $127-139$.

Munk R., 1968 a. - Über den Feinbau der Filterkammer der Kleinzikade Euscelidius variegatus Kbm. (Jassidae). Z. Zellforschung, 85, 210-224.

Munk R., 1968 b. - Die Richtungen des Nahrungsflusses im Darmtrakt der Kleinzikade Euscelidius variegatus Kbm. (Jassidae). Z.vgl. Physiol., 58, 423-428.

MUNK R., 1968 c. - Autoradiographische Untersuchungen des Transportes einiger Nahrungsbestandteile im Darmtrakt zweier Kleinzikaden : Eusclidius variegatus Kbm. (Jassidae) und Triecphora vulnerata Germ. (Cercopidae). Z. vgl. Physiol., 61, 129-136.

Salama H. S., Rizk A. M., 1969. - Composition of the honey dew in the mealy bug, Saccharicoccus sacchari. J. Inscct Physiol., 15, 1873-1875.

Saleh M., Salam A. H. S., 1971. - Main chemical components of the honeydew excreted by the vine mealy bug Planococcus vitis. J. Insect Physiol., 17, 1661-1663.

Schefer Immel V., 1957. - Über ein Massenauftreten der Zikade Adiocerus laminatus Flor. an Populus tremulus, mit einigen Bemerkungen zur Biologie und zum Honigtau der Art. Anz. Schädlingskd., 30, 165-169. 
Scheuner S., 1966. - Ein Beitrag zur Verbreitung der auf Fichte (Picea excelsa (L.) Karsten) lebenden honigtauliefernden Kienläuse (Homoptera, Cinarinae). Hercynia, 3, 359-373.

Scheurer S., 1969. - Materialien zur Verbreitung der auf Pinus silvestris L. lebenden Cinarinen im Gebiet der DDR. Hercynia, 6, 319-337.

Scheurer S., 1970. - Morphologische Merkmale der an Pinus silvestris gefundenen Cinarinen (Homoptera Lachnidae) im Bereich der Dübener Heide (DDR). Ann. Zool. (Warsaw), 28, 22-42.

Scheurer S., 1971. - Morphologische Studien an Cinara brauni Börner, 1940 (Homoptera. Lachnidae). Ann. Zool., (Warsaw), 28, 345-352.

Schmutterer H., 1953. - Aphiden und Cocciden als Honigtauerzeuger auf Laubhülzern. Z. angew. Ent., 34, 607-612.

Schmuttener H., 1958. - Die Honigtau-Erzeuger Mitteleuropas. Z. angew. Entomol., 42, 409-419.

Shearer J. W., 1976. - Effect of aggregations of aphids (Periphyllus sp.) on their size. Ent, exp. appl., 20, 179-182.

Singh Sidhu H., Patton R. L., 1970. - Carbohydrates and nitrogenous compounds in the honeydew of the mustard aphid, Lipaphis erysimi. J. Insect Physiol., 16, 1339-1348.

Sмiтн L. M., 1937. - Growth, reproduction, feeding, and wing development of the mealy plum aphid in relation to climatic factors. $J$. Agr. Research, 54, 345-364.

Srivastava P. N. Auclair J. L., 1962. - Characteristics of invertase from the alimentary canal of the pea aphid, Acyrthosiphon pisum (Harr.) (Homoptera, Aphididae). J. Insect Physiol., 8, 527-535.

Way M. J., Cammell M., 1970. - Aggregation behaviour in relation to food utilization by aphids. In : WATson A. ed., Animal populations in relation to their food resources (Symposium the British Ecological Soc. Aberdeen 1969) Blackwell Scientific Publications, Oxford + Edinburgh, $477 \mathrm{p}$.

Werer H., 1930. - Biologie der Hemipteren. Biologische Studienbücher, Verlag J. Springer, Berlin 543 p.

Wolf I. P., Eward W. H., 1955. - Carbohydrate composition of the honeydew of Coccus hesperidum L. Evidence for the existence of two new oligosaccharides. Arch. Bioch. and Biophys. 58, 365 .

Ziggler H., 1956. - Untersuchungen über die Leitung und Sekretion der Assimilate. Planta, (Berlin) 47, 447-500.

Ziegler H., 1975. - Nature of transported substances, 59-100. In : Zimmermanv M. H., Minburn I. A., eds. Transport in Plants I. Encyclopedia of Plant Physiology Vol. I Springer-Verlag Berlin, Heidelberg, New York, $535 \mathrm{p}$.

Ziegler H. J., Mittleh T. E., 1959. - Über den Zuckergehalt der Siebröhren bzw. Siebzellensäfte von Heracleum Mantegazzianum und Picea abies (L.) Karst. Z. Naturforsch., 14 b, 278-281.

ZimmermanN M. H, Ziegler H., 1975. - List of sugars and sugar alcohols in sieve-tube exudates, 180-503. In : Zimmermann M. H., Mrtburn J. A. eds. Transport in Plants I. Encycloperlia of Plant Physiology Vol. I Springer-Verlag Berlin, Heidelberg, New York $535 \mathrm{p}$. 